\title{
Impact of a Square Liquid Body on a Rigid Plate
}

\author{
Jian $\mathrm{Hu}^{1}$, Li-chao Liu ${ }^{1}$, Yu-hang Chen ${ }^{1}$, Peng Li ${ }^{2}$, Lei Guo ${ }^{1}$ and Shi-li Sun ${ }^{1 *}$ \\ ${ }^{1}$ College of Shipbuilding Engineering, Harbin Engineering University, Harbin, 150001, China \\ ${ }^{2}$ Guangzhou Marine Engineering Cooperation, Guangzhou, 510250, China
}

Keywords: Fluid Impact, VOF, Liquid Square, Free Surface Evolution.

\begin{abstract}
Based on the Finite Volume Method (FVM), the impact on a rigid plate by a free falling liquid body is investigated in this paper. The Volume of Fluid (VOF) technique is used to track the free surface. Based on the proposed technique, the impact of a free falling liquid body is simulated, and the effects of volume and initial height of the liquid body is analyzed.
\end{abstract}

\section{Introduction:}

The phenomenon of droplet impacting on solid surfaces widely exists in both nature and engineering systems. So, study of droplet impact with free surface is of great importance in a variety of different fields. For example, in agriculture the prevention of soil erosion due to rain plays an important role. It is very important for studying the mechanism leading to the erosion of a material due to liquid impingement, too. And in fluid field, the simulation of viscous free-surface is one of the most interesting and challenging problems.

Yang, Huang and Hammitt (1973) have studied the hydrodynamic phenomena during high-speed collision between liquid droplet and rigid plane [1]. Huang Bradford and Cushman (1982) have studied the raindrop impact phenomena using the Marker and Cell (MAC) method [2]. Rein (1993) has described and reviewed the phenomena of liquid drop impact, including bouncing, spreading and splashing on solid surfaces [3]. Li, Zhang, Guo and Lv (2014) measured the impact forces of the low-speed water droplet colliding on a light and tiny aluminum plate using a highly sensitive piezoelectric force transducer [4].

And in this paper, convergence of our method is proved firstly. Then, the effects of volume and initial height of the liquid body on the free surfaces evolution and the pressure distribution of the plate are analyzed. The impact force on the rigid plate is summarized.

\section{Governing Equations:}

Written in the differential form, the continuity equation reads:

$$
\frac{\partial \rho}{\partial t}+\nabla \cdot(\rho \mathbf{V})=0
$$

Where $\rho$ is the fluid density, $\mathbf{V}=(u, v, w)$ is the velocity. And for incompressible fluid, it can be written as:

$$
\nabla \cdot \mathbf{V}=0
$$

The continuity equation states that, in any steady state process, the rate at which mass enters a system is equal to the rate at which mass leaves the system.

Navier-Stokes (N-S) equations can be written as:

$$
\frac{D(\rho \mathbf{V})}{D t}=\mu \nabla^{2} \mathbf{V}-\nabla P+\mathbf{g}+\mathbf{f}_{\sigma}+\mathbf{S}
$$

Where, ${ }^{\mu}$ is the mixed dynamic coefficient of viscosity, $P$ is pressure, $\mathbf{g}=(0,0,-g)$ is 
gravity, $\mathbf{f}_{\boldsymbol{\sigma}}=\sigma \kappa$ is the surface tension, $\mathbf{S}=\left(S_{x}, S_{y}, S_{z}\right)$ is the source item. Eq. 3 describes the force balance at a given point within a fluid. Solutions of the Navier-Stokes equations for a given physical problem must be sought with the help of calculus.

VOF method is based on the idea of a so-called volume fraction function $F$. It is a scalar function, defined as the integral of a fluid's characteristic function in the control volume, namely the volume of a computational grid cell. When a cell is empty with no traced fluid inside, the value of $F$ is zero; when the cell is full, the value of $F$ is one; and when there is a fluid interface in the cell, $0<F<1 \quad$ [5].

$$
F(\mathbf{x}, t)=\left\{\begin{array}{cl}
1 & \text { full of fluid } \\
0<F<1 & \text { air and fluid } \\
0 & \text { full of air }
\end{array} .\right.
$$

Transport equation of $F$ can be written as:

$$
\frac{D F}{D t}=0
$$

It stands for transportation laws of volume fraction function $F$.

\section{Discretization of Governing Equations}

The discrete continuity equation can be written as:

$$
\sum_{n f=1}^{n} \mathbf{u}_{f} \cdot \mathbf{A}_{f}=0
$$

Where $\mathbf{u}_{f}$ is velocity of the interface, $\mathbf{A}_{f}$ is area of the interface.

For the discrete N-S equations, the deferred correction is used. Based on the deferred correction, the convective flux of the interface can be expressed as sum of the first-order upwind-style convective flux and a source term. In the N-S equations, the discrete convective term and the discrete diffusion term are obtained by the first-order upwind scheme and the central differencing scheme respectively.

The discrete N-S equations can be written as:

$$
A_{P} \phi_{P}=A_{E} \phi_{E}+A_{W} \phi_{W}+A_{N} \phi_{N}+A_{S} \phi_{S}+b_{P}+b_{d c}
$$

Where, $A$ is the coefficient which is obtained based on the first-order upwind scheme, $b_{P}=S_{m} \Delta V$ is the source item, $b_{d c}$ is the source item which is caused by the deferred correction. And the subscript refers to location of the interface.

In addition, the fully implicit scheme is used for the instantaneous item, which can guarantee computational stability even when the time step is large. The discrete instantaneous item always contains unknown quantities at the new time step and the old time step. To evaluate the equation we always use a weighting parameter $f$ between 0 and 1 . In the fully implicit scheme, the value of $f$ is set to 1 , then both sides of the equation contain unknown quantities at the new time step.

\section{Convergence Validation of Pesent Numerical Simulation}

At the initial time, a square liquid body is confined above the plate. The square liquid body is $0.7 \mathrm{~m}$ wide and $0.7 \mathrm{~m}$ high. We define height of the liquid body h1, the initial falling-height $\mathrm{h} 2$. Then, 
$\mathrm{h} 1$ is $0.15 \mathrm{~m}$. And the computational domain is $2 \mathrm{~m}$ long and $1 \mathrm{~m}$ high, shown in Fig. 1. Four upper, bottom, left and right boundaries of the computational domain are all no-slip walls. The initial pressure depends on the static pressure distribution. And initial pressure on the wall is defined zero.

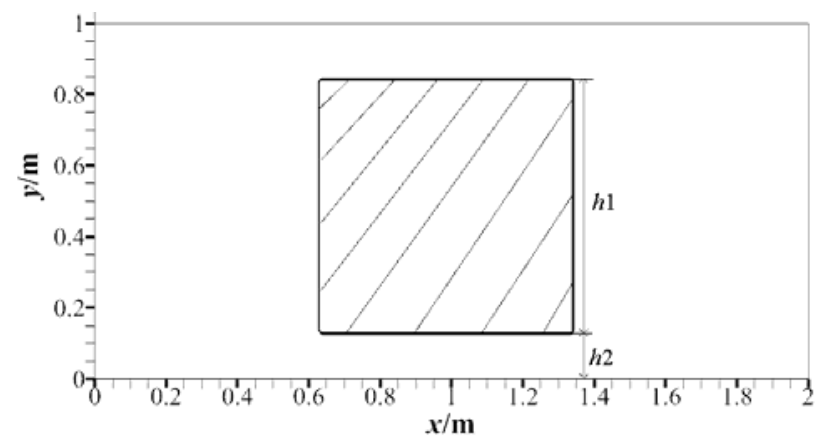

Figure 1. Numerical model for convergence validation

Table.1 Different grid numbers

\begin{tabular}{|c|c|c|c|}
\hline & Row grid number & Column grid number & Total grid number \\
\hline 1 & 200 & 100 & 20000 \\
\hline 2 & 240 & 120 & 28800 \\
\hline 3 & 280 & 140 & 39200 \\
\hline 4 & 360 & 180 & 64800 \\
\hline
\end{tabular}

To verify convergence of the numerical simulation, four different grids numbers for the numerical model is used, as in Table 1. Profiles of the free surface in the impacting process changes very little with different grid number, as shown Fig. 2. The developments of the free surface under different grid numbers prove the convergence of our method.

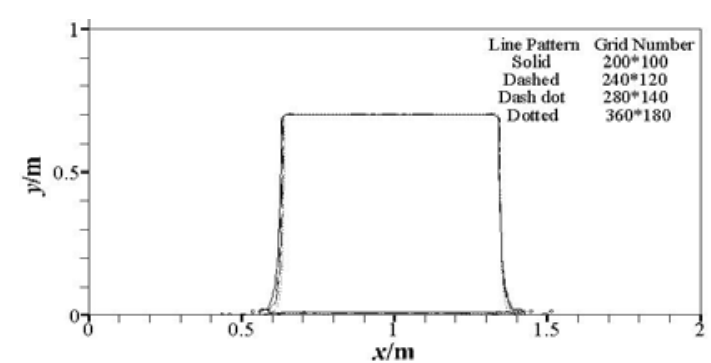

(a) $t=0.17 \mathrm{~s}$

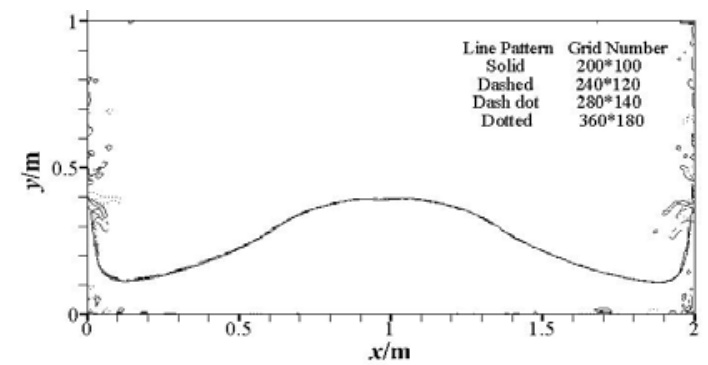

(b) $t=0.37 \mathrm{~s}$

Figure 2. Profile of the free surface under different grid numbers when $t=0.17 \mathrm{~s}$ and $0.37 \mathrm{~s}$ respectively

\section{Results and Discussions}

\section{Impact on the Plate of the Liquid Body at Various Initial Height $h_{1}$}

To observe the differences when the square liquid body falls from different heights, this article simulates the phenomena with varying height for $0.1 \mathrm{~m}$ (see Fig. 3) and $0.4 \mathrm{~m}$ respectively. The square liquid is $0.4 \mathrm{~m}$ high and $0.4 \mathrm{~m}$ wide. The computational domain is $3 \mathrm{~m}$ wide and the height is $0.6 \mathrm{~m}$ and $0.9 \mathrm{~m}$, corresponding to different initial height of the liquid square.

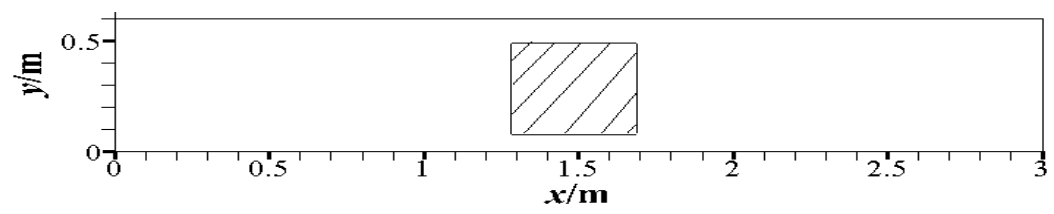

Figure 3. Numerical model when the drop-height is $0.1 \mathrm{~m}$

Pressure coefficient $C p$ is defined as: 


$$
C_{p}=\frac{P}{\rho_{w} g H_{0}} .
$$

Where $H_{0}$ is width of the liquid column, which is constant as $0.4 \mathrm{~m}$ in the all the following simulations.

The liquid body's falling makes the surrounding air to rotate violently. This causes the low-pressure areas at the corners of the liquid body. Shown in Fig. 4, at the moment when the liquid touches the plate, great pressure is caused by the impacting. The peak of the pressure distribution curve is the specific reflection. The higher the drop-height is, the greater the impacting pressure is. As time goes by, the liquid spreads out, and the pressure distribution is more uniform. When the liquid touch the side walls with a velocity, pressure of both sides have an upsurge.

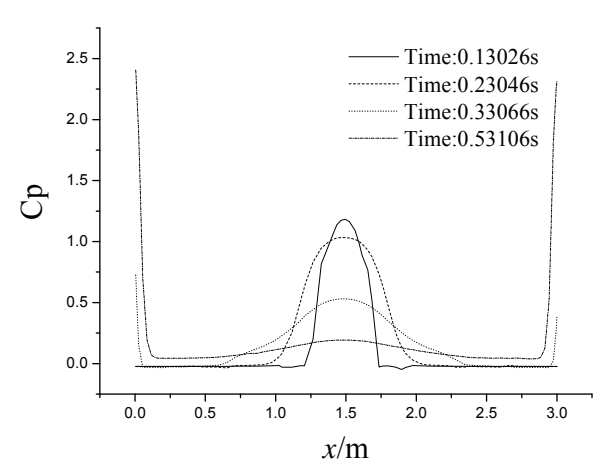

(a) $h_{2}=0.1 \mathrm{~m}$

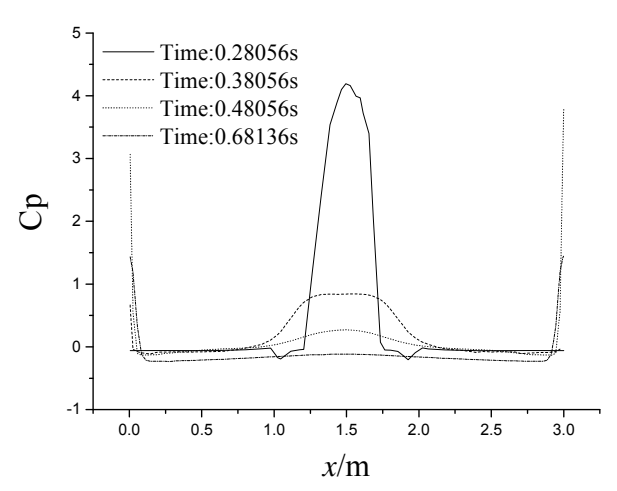

(b) $h_{2}=0.4 \mathrm{~m}$

Figure 4. Pressure distribution with time when the initial drop-height is $0.1 \mathrm{~m}$ and $0.4 \mathrm{~m}$

\section{Impact on the Plate of the Liquid Body at Various $h_{2}$}

Using the same computational domain and keeping $\mathrm{h} 1$ of $0.1 \mathrm{~m}$, the impacts of the liquid at various time instant as $\mathrm{h} 1=0.4 \mathrm{~m}, 0.5 \mathrm{~m}, 0.6 \mathrm{~m}$ and $0.7 \mathrm{~m}$ are shown Fig. 5(a), 5(b), 5(c) and 5(d) respectively. From Fig. 5(a), it is found the pressure as $\mathrm{h} 1=0.4 \mathrm{~m}$ at the center of the rigid plate peaks at $\mathrm{t}=0.13026$. Comparing Figs $5(\mathrm{a}), 5(\mathrm{~b}), 5(\mathrm{c})$ and $5(\mathrm{~d})$, it is found that the pressure peak at the plate center is delayed in time phase and increased in magnitude. Generally, the pressure variation with respect to $h 1$ is not as obvious as that with respect to $h 2$.

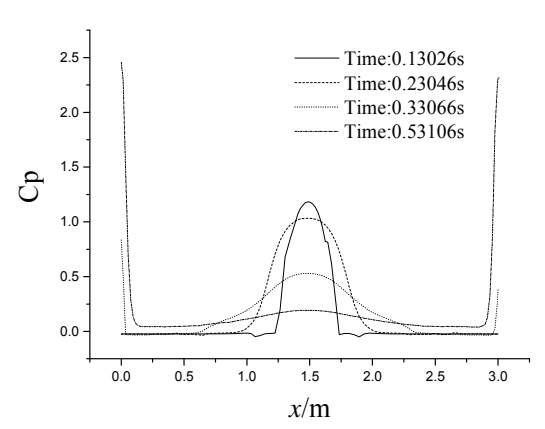

(a) $h_{1}=0.4 \mathrm{~m}$

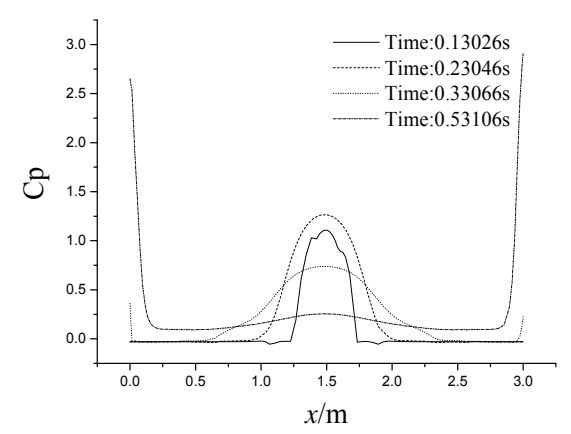

(b) $h_{1}=0.5 \mathrm{~m}$ 


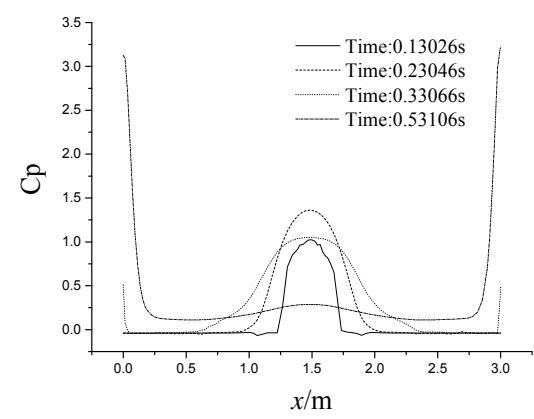

(c) $h_{1}=0.6 \mathrm{~m}$

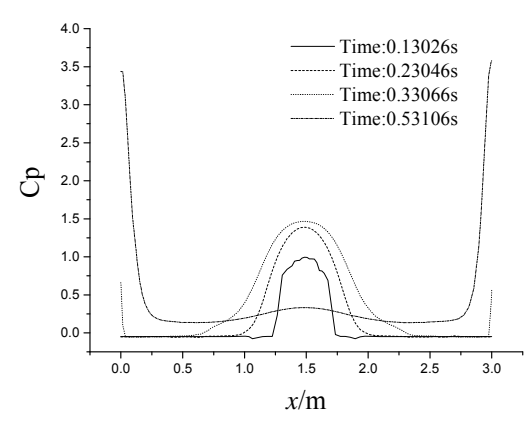

(d) $h_{1}=0.7 \mathrm{~m}$

Figure 5. Pressure distribution with time when the body height is $0.4 \mathrm{~m}, 0.5 \mathrm{~m}, 0.6 \mathrm{~m}$ and $0.7 \mathrm{~m}$ respectively

\section{Conclusions}

In the present paper, a robust numerical code is developed and applied in the simulation of liquid impact. The convergence study is performed with respect to the grid number. The results showed great conformity for the free surface. Then the impact of a liquid square on a rigid plate is studied, and the effects of the initial size and volume of the liquid on the impact are analyzed.

\section{Acknowledgement}

The authors are grateful for the support of the National Natural Science Foundation of China (Grant Nos. $51678045,51579052)$.

\section{References}

[1] Y. C. Huang, F. G. Hammitt, W. J. Yang, Hydrodynamic Phenomena During High-Speed Collision Between Liquid Droplet and Rigid Plane, J. Fluids. 95 (1973) 276-292.

[2] C. Huang, J. M. Bradford, J. H. Cushman, A Numerical Study of Raindrop Impact Phenomena: The Rigid Case, SSSAJ. 46 (1982) 14-19.

[3] M. Rein, Phenomena of liquid drop impact on solid and liquid surfaces, Fluid Dynamics Research. 12 (1991) 61-93.

[4] J. Y. Li, B. Zhang, P. H. Guo, Q. Lv, Impact force of a low speed water droplet colliding on a solid surface, Journal of Applied Physics. 116 (2014) 214903.

[5] M. Seifollahi, E. Shirani, N. Ashgriz, An improved method for calculation of interface pressure force in PLIC-VOF methods, Europran Journal of Mechanics B/Fluids. 27 (2008) 1-13. 